\title{
Rayleigh-Brillouin Scattering to Determine One-Dimensional Temperature and Number Density Profiles of a Gas Flow Field
}

James A. Lock

Cleveland State University, j.lock@csuohio.edu

Richard G. Seasholtz

W. Trevor John

Follow this and additional works at: https://engagedscholarship.csuohio.edu/sciphysics_facpub

Part of the Physics Commons

How does access to this work benefit you? Let us know!

\section{Publisher's Statement}

This paper was published in Applied Optics and is made available as an electronic reprint with the permission of OSA. The paper can be found at the following URL on the OSA website: http://www.opticsinfobase.org/ao/abstract.cfm?URI=ao-31-15-2839. Systematic or multiple reproduction or distribution to multiple locations via electronic or other means is prohibited and is subject to penalties under law.

\section{Original Citation}

Lock, James A., Richard G. Seasholtz, and W. Trevor John. "Rayleigh-Brillouin Scattering to Determine One-Dimensional Temperature and Number Density Profiles of a Gas Flow Field." Applied Optics 31 (1992): 2839-2848.

\section{Repository Citation}

Lock, James A.; Seasholtz, Richard G.; and John, W. Trevor, "Rayleigh-Brillouin Scattering to Determine OneDimensional Temperature and Number Density Profiles of a Gas Flow Field" (1992). Physics Faculty Publications. 87.

https://engagedscholarship.csuohio.edu/sciphysics_facpub/87

This Article is brought to you for free and open access by the Physics Department at EngagedScholarship@CSU. It has been accepted for inclusion in Physics Faculty Publications by an authorized administrator of EngagedScholarship@CSU. For more information, please contact library.es@csuohio.edu. 


\title{
Rayleigh-Brillouin scattering to determine one-dimensional temperature and number density profiles of a gas flow field
}

\author{
James A. Lock, Richard G. Seasholtz, and W. Trevor John
}

\begin{abstract}
Rayleigh-Brillouin spectra for heated nitrogen gas were measured by imaging the output of a Fabry-Perot interferometer onto a CCD array. The spectra were compared with the theoretical 6-moment model of Rayleigh-Brillouin scattering convolved with the Fabry-Perot instrument function. Estimates of the temperature and a dimensionless parameter proportional to the number density of the gas as functions of position in the laser beam were calculated by least-squares deviation fits between theory and experiment.
\end{abstract}

\section{Introduction}

The quasi-elastic scattering of light by an atomic or molecular gas is known as Rayleigh-Brillouin scattering. ${ }^{1-3}$ At a low gas density the frequency of the scattered light is Doppler shifted by the thermal motions of the individual gas molecules. At a high gas density the frequency shift is produced by the interaction with various collective motions of the molecules, specifically sound waves and thermal diffusion modes. If $\lambda_{0}$ and $\omega_{0}$ are the wavelength and angular frequency of the incident light, the frequency shift of the scattered light and the gas density may be parameterized by the dimensionless quantities:

$$
\begin{aligned}
& x=\frac{\Delta \omega}{\kappa}\left(\frac{m}{2 k T}\right)^{1 / 2}, \\
& y=\frac{\mathscr{N}}{\kappa \eta}\left(\frac{m k T}{2}\right)^{1 / 2},
\end{aligned}
$$

where

$$
\Delta \omega=\omega_{\text {scattered }}-\omega_{0}
$$

the wave number associated with the scattering

\footnotetext{
James A. Lock is with the Department of Physics, Cleveland State University, Cleveland, Ohio 44115; Richard G. Seasholtz is with NASA Lewis Research Center, Cleveland, Ohio 44135; W. Trevor John is with Calspan Corporation, NASA Lewis Research Center, Cleveland, Ohio 44135.

Received 27 December 1990.

0003-6935/92/152839-10\$05.00/0.

(C) 1992 Optical Society of America.
}

momentum transfer is

$$
\kappa=\frac{4 \pi}{\lambda_{0}} \sin \frac{\alpha}{2}
$$

where $\alpha$ is the scattering angle; $k$ is Boltzmann's constant, and $\mathscr{N}, \eta, m$, and $T$ are the number density, shear viscosity, molecular mass, and temperature of the gas, respectively.

For gases containing a single species, it has been shown that the normalized scattered intensity per molecule $S$ is a function of $x$ and $y$ alone. ${ }^{4}$ A number of kinetic theory models for $S(x, y)$ have been developed for single-species atomic ${ }^{5-8}$ and molecular ${ }^{9-12}$ gases and compared with experimental data. ${ }^{13-19}$ The 6-moment model of Ref. 11 is the best model that is currently available for the description of RayleighBrillouin scattering by a single-species gas. ${ }^{20,21}$ By using various kinetic theory models, RayleighBrillouin scattering has now begun to be employed as a diagnostic technique to determine the temperature and number density of gases in combustion ${ }^{22,23}$ and remote sensing situations. ${ }^{24-27}$

With this technique the temperature of a gas sample is determined by scaling the parameter $x$ of Eq. (1) to the measured frequency spectrum. The number density of the gas is determined from the shape of the frequency spectrum by the density parameter $y$ of Eq. (2). This technique represents a novel way of measuring the gas density, since the absolute magnitude of the scattered light intensity (which is usually employed in Rayleigh scattering determinations of density) is not measured. When using this technique, great care must be taken in 
measuring and analyzing the scattered spectrum since the temperature is a rapidly varying function of $x$ (i.e., $\propto 1 / x^{2}$ ). This rapid variation is potentially capable of producing a large uncertainty in the computed temperature if even a moderate uncertainty exists in the experimental frequency spectrum. The best results for obtaining the density should occur where the spectral shape is most sensitive to changes in $y$. This occurs in the so-called kinetic regime for which $y \sim 1$.

A Fabry-Perot interferometer may be employed either in the scanning mode or in the static mode to measure the Rayleigh-Brillouin spectrum. In the scanning mode the frequency spectrum is obtained by varying the separation between the interferometer's etalon plates. By positioning a single detector in the focal plane of the fringe-forming lens at the center of the interference pattern, the spectrum is obtained for one location within the gas sample. If the instrument is used in the static mode with a detector array at the focal plane, spectra can be measured throughout a plane section of the gas sample. The frequency spectrum in this case is given by the radial dependence of the intensity of the Fabry-Perot fringe rings.

The Fabry-Perot imaging configuration has been used to obtain temperature and velocity profiles of thermospheric winds during geomagnetic storms. ${ }^{28-30}$ We describe an experiment that measures onedimensional temperature and number density profiles of a gas flow field by using Rayleigh-Brillouin scattering and the Fabry-Perot interferometer in the imaging configuration. The ultimate goal of this work is the development of a two-dimensional single-pulse combustion diagnostic.

The present experiment, by assessing the practicality of the imaging Rayleigh-Brillouin scattering technique, serves as an initial step toward this goal. Statistical errors in temperature measurements for this application are discussed in Appendix A.

\section{Fabry-Perot Interferometer in the Imaging Configuration}

Consider the geometry of Fig. 1. A ribbon laser beam of thickness $t$ is formed by a cylindrical lens and passes through a gas sample. An element of illuminated volume $d V_{i}=t\left(d A_{i}\right)$ centered at the coordinate $\mathbf{r}_{i}$ is in the focal plane of a lens that is a distance $f_{1}$ away. Molecules within the illuminated volume scatter light through the angle $\alpha$ into the lens with the angular frequency shifted by $\Delta \omega$. The light passes through the lens and into the Fabry-Perot interferometer whose intensity transmission function is $T(\omega, \theta)$ where

$$
\tan \theta=r_{i} / f_{1}
$$

Light leaving the interferometer passes through a second lens of focal length $f_{2}$ and is imaged onto a CCD array. The volume element $d V_{i}$ is imaged onto a single CCD pixel of area $d A_{0}$ centered at the position

$$
\mathbf{r}_{0}=M \mathbf{r}_{i}
$$

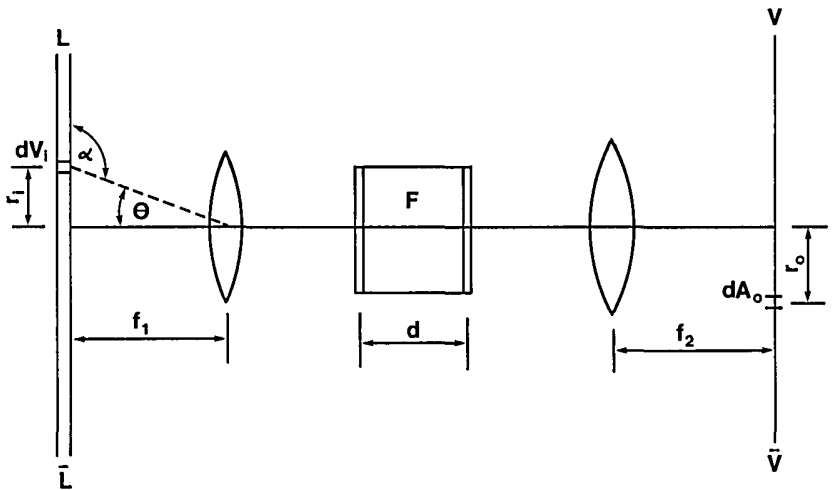

Fig. 1. Fabry-Perot interferometer used in the imaging mode. $\mathrm{L} \overline{\mathrm{L}}$ is the laser beam, $\mathrm{F}$ is the interferometer whose etalon plate spacing is $d$, and $V \bar{V}$ is the CCD array. The scattering volume $d V_{i}$ is imaged onto the pixel of area $d A_{0}$ located at the coordinate $\vec{r}_{0}$ on the array.

where the magnification of the imaging system is

$$
M=\frac{-f_{2}}{f_{1}} .
$$

If the counting efficiency of the CCD pixel is $\epsilon$, the number of photons per second of all frequencies that are counted by the CCD element is

$$
\begin{aligned}
\mathscr{R}\left(\mathbf{r}_{0}\right)=I_{\text {laser }}\left(\mathbf{r}_{i}\right)\left[\frac{d \sigma}{d \Omega}(\alpha)\right] \mathscr{N}\left(\mathbf{r}_{i}\right)\left(\Delta \Omega_{i}\right) & \frac{t\left(d A_{0}\right)}{M^{2}} \frac{\epsilon}{\omega_{0} \hbar} \\
& \times \int_{-\infty}^{\infty} \mathrm{d} x S(x, y) T\left(\omega, \frac{r_{0}}{f_{2}}\right),
\end{aligned}
$$

where $I_{\text {laser }}\left(\mathbf{r}_{i}\right)$ is the laser intensity at $\mathbf{r}_{i},(d \sigma / d \Omega)(\alpha)$ is the Rayleigh scattering differential cross section per molecule at the scattering angle $\alpha, \mathscr{N}\left(\mathbf{r}_{i}\right)$ is the number density of molecules at $\mathbf{r}_{i}, \Delta \Omega_{i}$ is the solid angle of lens 1 subtended at the scattering volume $d V_{i}, \hbar$ is Planck's constant divided by $2 \pi$, and $S(x, y)$ is the fraction of the total scattered intensity between the frequencies corresponding to the parameters $x$ and $x+\mathrm{d} x$. In Eq. (8) we have assumed that $I_{\text {laser }}$ and $\mathscr{N}$ are constant throughout the volume $d V_{i},(d \sigma /$ $d \Omega)(\alpha)$ is constant throughout the solid angle $\Delta \Omega_{i}$, the counting efficiency, of the CCD element is frequency independent, ' $\Delta \omega$ is small compared with $\omega_{0}$, and $r_{0} \ll f_{2}$. Collecting all the constants together, we may rewrite Eq. (8) as

$$
\mathscr{R}\left(\mathbf{r}_{0}\right)=K \int_{-\infty}^{\infty} \mathrm{d} x S(x, y) T\left(\omega, \frac{r_{0}}{f_{2}}\right) .
$$

The transmission function of the Fabry-Perot interferometer is ${ }^{31,32}$

$$
T(\omega, \theta)=\left[1+\frac{\sin ^{2}\left(\frac{\omega d \cos \theta}{c}\right)}{\sin ^{2}\left(\frac{\pi}{2 \mathscr{I}}\right)}\right]^{-1},
$$


where $c$ is the velocity of light, $d$ is the etalon plate spacing, and $\mathscr{F}$ is the instrument's finesse.

Consider the transmission function for the laser frequency $\omega_{0}$ for small $\theta$ where

$$
\cos \theta \approx 1-\frac{r_{0}^{2}}{2 f_{2}^{2}} .
$$

The nonlinearity of the cosine function implies that each instrument function fringe is asymmetric with respect to its center; the frequency shift scale parameterized by the quantity $x$ of Eq. (1) is a nonlinear function of pixel position, and Eq. (9) does not reduce to a convolution integral. ${ }^{33}$ However, to assess the importance of the nonlinearity in the present experiment we let $R_{0}$ be the position of the center of a particular Fabry-Perot fringe on the CCD array with respect to the center of the Fabry-Perot fringe pattern for the laser frequency $\omega_{0}$, and we measure positions on the CCD array with respect to $R_{0}$ (i.e., $r_{0}=R_{0}+u$ ). In the conditions of the present experiment $\omega_{0} \approx 4 \times 10^{15} \mathrm{rad} / \mathrm{s}, \Delta \omega \approx 6 \times 10^{9} \mathrm{rad} / \mathrm{s}$ (i.e., the thermal half-width at half-maximum of the Rayleigh-Brillouin spectrum), $R_{0} \approx 4 \mathrm{~mm}$ at a typical fringe, $\delta=0.02 \mathrm{~mm}$ is the half-width at halfmaximum of the instrument function fringe, $d \approx 20$ $\mathrm{mm}, f_{2} \approx 500 \mathrm{~mm}$, and $u<0.09 \mathrm{~mm}$ for the gas spectrum corresponding to that fringe. For these conditions, although there is substantial nonlinearity from fringe to fringe in the Fabry-Perot pattern, the nonlinearity across a single fringe is found to be only a small effect, and the instrument function may be approximated to within $\sim 2 \%$ accuracy for a single fringe by

$$
T\left(\omega_{0}+\Delta \omega, \frac{r_{0}}{f_{2}}\right)=\left[1+\frac{\sin ^{2}\left(\frac{\omega_{0} d R_{0} u}{f_{2}^{2} c}-\frac{d \Delta \omega}{c}\right)}{\sin ^{2}\left(\frac{\omega_{0} d R_{0} \delta}{f_{2}^{2} c}\right)}\right]^{-1}
$$

With this approximation to the instrument function, Eq. (9) for a single fringe may be written as the convolution integral

$$
\mathscr{R}(\xi) \approx K \int_{-\infty}^{\infty} \frac{\mathrm{d} x S(x, y)}{1+\frac{\sin ^{2}[w(\xi-x)]}{\sin ^{2}(w \beta \delta)}},
$$

where

$$
\begin{aligned}
& \beta=\frac{\omega_{0} R_{0}}{\kappa f_{2}^{2}}\left(\frac{m}{2 k T}\right)^{1 / 2}, \\
& w=\frac{\kappa d}{c}\left(\frac{2 k T}{m}\right)^{1 / 2}, \\
& \xi=\beta u .
\end{aligned}
$$

This convolution approximation applied to only one fringe at a time considerably simplifies the analysis of the single fringe. ${ }^{34}$

\section{Experimental Procedure}

A schematic diagram of the apparatus is shown in Fig. 2. An étalon was used with a Model 95 Lexel argon-ion laser to provide the single axial mode $\left(\lambda_{0}=514.5 \mathrm{~nm}\right)$ that is needed for spectral measurements. The laser output was $0.4 \mathrm{~W}$, and the beam was vertically polarized. A cylindrical lens system was used to focus the beam into the center of the $38-\mathrm{cm}$ diam stainless-steel scattering chamber. The resulting ribbon beam had a thickness of $100 \mu \mathrm{m}$ and a height of $1 \mathrm{~mm}$. The optics that were used to form the ribbon beam consisted of one spherical lens and two cylindrical lenses. The spherical lens and one cylindrical lens (oriented with its axis vertical) were used to set the beam thickness $t$ to $100 \mu \mathrm{m}$ at the center of the field of view inside the scattering chamber. The spherical lens and second cylindrical lens (axis horizontal) were used to set the beam height to $1 \mathrm{~mm}$. Parasitic light was controlled with baffles located in the chamber, and the beam was terminated in a light trap.

A portion of the laser beam was coupled into an optical fiber through a manually controlled shutter. The output of the fiber was directed into the chamber and illuminated the field of view of the light collection system. This provided an approximately spatially uniform light source at the laser frequency and was used to measure the instrument function of the interferometer.

Light scattered at $90^{\circ}$ was collected through a port in the chamber and collimated by an $f / 5.6,500-\mathrm{mm}$ focal-length telephoto lens. The input aperture of the interferometer was $70 \mathrm{~mm}$, which resulted in an effective $f$-number of 7.1 for the collection of the Rayleigh scattered light. After passing through the Fabry-Perot interferometer (Burleigh Model RC110 , mirror reflectance of $90 \%$ ), the light was focused by a second $500-\mathrm{mm}$ focal length lens onto the $384 \times$ 576 pixel array of a Photometrics Star I cooled CCD camera. The light scattered from the ribbon beam in the scattering chamber was thus imaged onto the CCD array with the image modulated by the transmis-

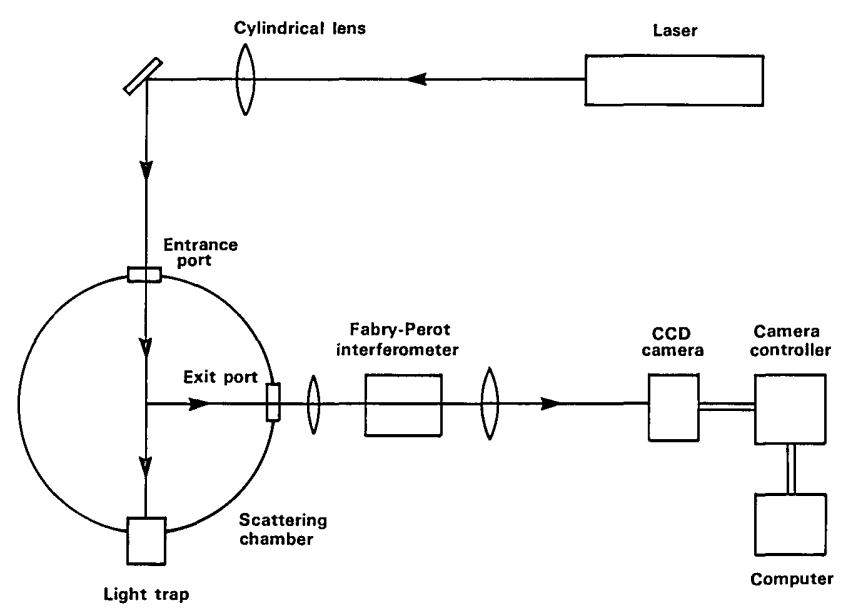

Fig. 2. Experimental arrangement for Rayleigh-Brillouin scattering using the Fabry-Perot interferometer in the imaging mode. 
sion function of the Fabry-Perot interferometer. Each pixel was $23 \mu \mathrm{m} \times 23 \mu \mathrm{m}$ square. The exposure time could be set $(0.1 \mathrm{~s}$ to hours); thus the scattered light could be integrated for sufficient time to achieve a satisfactory signal-to-noise ratio. The CCD images were digitized to $12 \mathrm{bits} /$ pixel by the camera controller and transferred to a Compaq 386/33 computer for storage.

A gas flow field was set up in the scattering chamber as shown in Fig. 3. A length of stainless-steel tubing of 2.1-mm inner diameter and 3.2-mm outer diameter was connected to an external tank of nitrogen. When nitrogen gas was blown gently through the tubing, the end of the tubing in the scattering chamber acted as a nitrogen gas jet. The tubing could be resistively heated so that the nitrogen leaving the jet had a temperature of up to $600 \mathrm{~K}$. The jet was $7 \mathrm{~mm}$ below the ribbon laser beam whose height was $1 \mathrm{~mm}$. An exit port $19 \mathrm{~mm}$ above the gas jet was fitted with a thermocouple to monitor the gas temperature and was connected to a vacuum pump to collect and remove the heat that was generated by the hot gas. The portion of the flow field sampled by the laser beam thus consisted of the gas in the chamber at ambient temperature and pressure and the localized region of heated gas immediately above the gas jet. With the imaging system of unit magnification, the CCD camera recorded $13.25 \mathrm{~mm}$ of this flow field along the length of the laser beam. A second thermocouple was placed $46 \mathrm{~mm}$ downbeam from the gas jet below the laser beam to measure the ambient temperature of the scattering chamber. The gas pressure within the chamber was also monitored, and the scattered spectrum was taken for both heated and unheated nitrogen leaving the gas jet.

Because the temperature is a rapidly varying function of the scaling of the frequency shift $x$ through Eq. (1), the instrument function fringes must be extremely stable and the fringe half-width at halfmaximum $\delta$ must be known accurately. As a test of

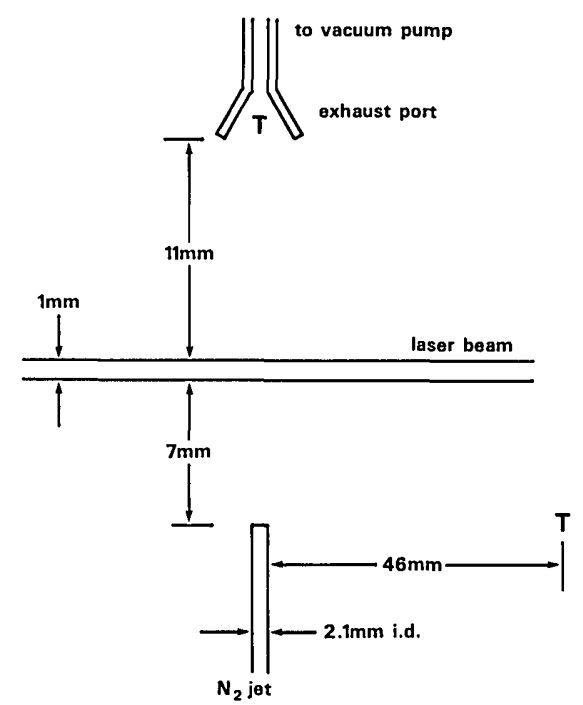

Fig. 3. Flow-field geometry inside the scattering chamber. The two thermocouples are at location 'I'. the stability of both the interferometer and the counting statistics of the CCD camera, exposure times from 10 to $300 \mathrm{~s}$ were examined for both the Fabry-Perot instrument function and the RayleighBrillouin frequency spectrum for unheated nitrogen. On a time scale of $\sim 10 \mathrm{~s}$ there were small temperature fluctuations within the scattering chamber that produced shifts in the fitted locations of the centers of the Fabry-Perot instrument function fringes of approximately \pm 0.1 pixel. It was found that integrating over a number of these shifts for longer exposure times did not widen the Fabry-Perot instrument function fringes seriously or overestimate the temperatures calculated from the gas spectra. It was also found that the temperatures derived from the gas spectra at atmospheric pressure showed a certain degree of variation until the exposure time was $\sim 50 \mathrm{~s}$. In addition the étalon plate alignment also had a small drift for exposures over $300 \mathrm{~s}$. As a result, for this first test of the feasibility of the Fabry-Perot imaging system, a compromise exposure time of $100 \mathrm{~s}$ (incident energy $\approx 40 \mathrm{~J}$ ) was chosen. The interferometer, imaging lens, and CCD camera were aligned so that the counting rate for each Fabry-Perot fringe was the same to both the left and right of the center of the fringe pattern. The center of the fringe pattern was positioned so that there were five or six usable fringes to each side of center. The central fringe was usually sufficiently wide and sufficiently nonlinear so as to be unusable for temperature and gas density determinations. An experimental run consisted of exposing the instrument function once and the scattering from the heated gas twice for each of seven separations of the interferometer etalon plates. Typical spectra are shown in Fig. 4.

\section{Data Analysis and Results}

Both the instrument function and the gas spectrum CCD images were preprocessed in the following way. The data from 15 rows of CCD pixels along the image of the laser beam were averaged to increase the signal-to-noise ratio. These 15 rows represented the central one-third of the laser beam. We felt that using more than 15 CCD rows would begin to show the effect of the curvature of the Fabry-Perot fringes, thus degrading the obtained spectra. The average dark count, which varied by only $\pm 0.5 \%$ across the CCD array, was subtracted from each pixel in the averaged array. The data for each individual fringe in the pattern were then put into separate 32-element arrays. An array of 32 elements was found to contain the entire frequency spectrum for each fringe examined. In addition the separation between adjacent fringes was always larger than 32 pixels so that each array contained scattering information from only one fringe.

In preparation for the convolution of the theoretical gas spectra the instrument function for each individual fringe in the experimental run was fitted separately to the Fabry-Perot transmission function $T\left(\omega_{0}, \theta\right)$ of Eq. (12) for $\Delta \omega=0$ through a least-squares 

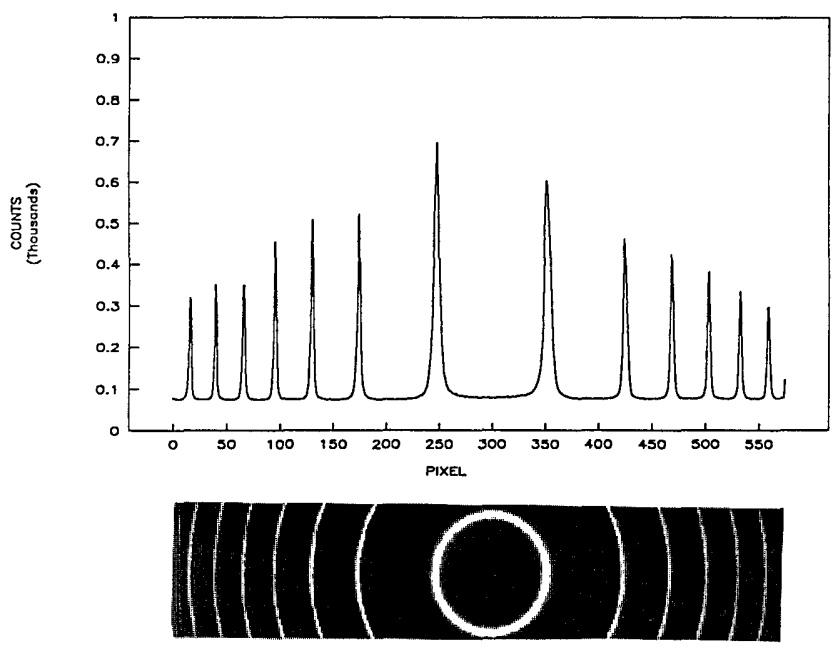

(a)
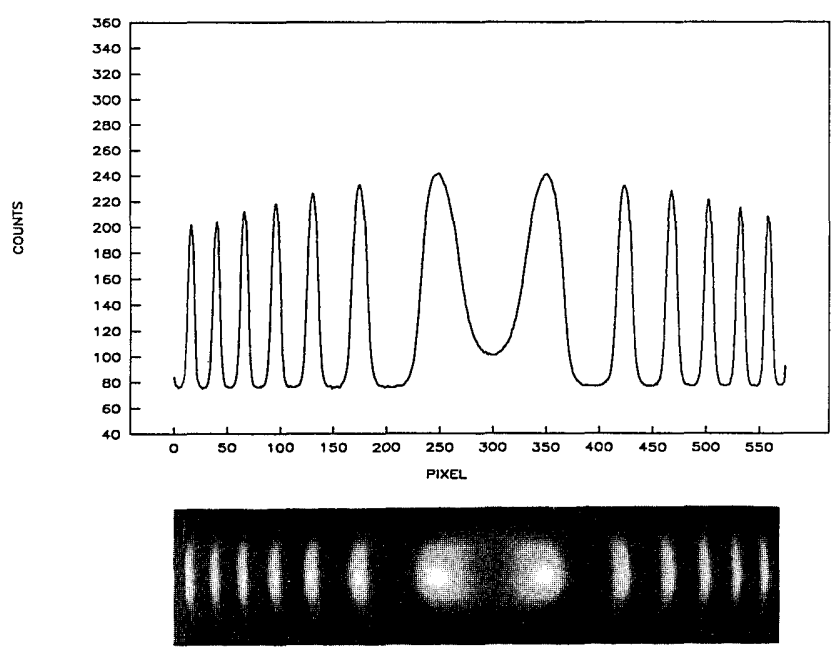

(b)

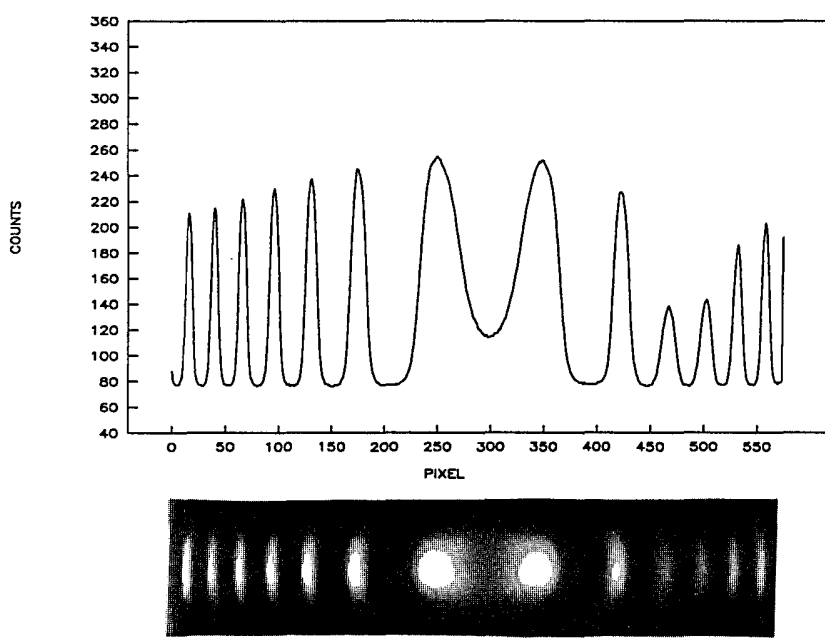

(c)

Fig. 4. Typical spectra for (a) the Fabry-Perot interferometer instrument function, (b) Rayleigh-Brillouin scattering from roomtemperature nitrogen gas, and (c) Rayleigh-Brillouin scattering from the elevated temperature flow field. deviation iterative calculation. The three-parameter fit produced an estimate for the height of the fringe, the distance of the peak of the fringe from the center of the Fabry-Perot ring pattern, and the half-width at half-maximum of the fringe. These last two quantities $R_{0}$ and $\delta$ were used as input for the theoretical gas spectrum convolution.

The normalized scattering function $S(x, y)$ that is used in the convolution of Eq. (13) was calculated by using the 6-moment model of Ref. 11. This model, although relatively accurate, is only an approximation to the actual scattering situation. ${ }^{12,20}$ Thus, before using this model with the frequency spectra of the present experiment, the accuracy of the 6-moment model was evaluated. In previous experimental verifications of the 6-moment model, the temperature and density of the illuminated gas were measured at the time the scattered light spectrum was taken. The verification consisted of visually comparing the 6-moment model for the values of $x$ and $y$ that correspond to the known temperature and density with the experimental data. In each case the convolved theoretical gas spectrum gave the visual appearance of fitting the experimental data quite well. In our evaluation of the accuracy of the 6-moment model we took a different point of view. For 35 sets of experimental data of $\mathrm{N}_{2}$ at four different pressures, using the Fabry-Perot interferometer in the scanning mode, we found the values of $x$ and $y$ that produced the best least-squares deviation fit to the experimental data. Thus we ascertained not how well the actual temperature and density inserted into the 6-moment model fit the experimental data but rather what temperature and density fit it the best.

The details of our evaluation when using the scanning interferometer $\mathrm{N}_{2}$ data (i.e., point measurements rather than imaging measurements) are as follows. The inputs to the 6-moment model are values of $x$ and $y$, the temperature dependence of the shear viscosity and the thermal conductivity of the gas, the ratio of the shear viscosity to the bulk viscosity, and the contribution to the specific heat provided by the internal degrees of freedom of the gas molecules. We parameterized the shear viscosity ${ }^{35}$ and thermal conductivity ${ }^{36}$ by

$$
\begin{gathered}
\eta(T)=170.7(T / 283.9)^{0.739} \quad \mu \mathrm{P}, \\
\Lambda(T)=58.27(T / 277.4)^{0.884} \quad \mu \mathrm{cal} / \mathrm{cm} \mathrm{s} \mathrm{K,}
\end{gathered}
$$

respectively. The ratio of viscosities employed was $1.367,{ }^{10}$ and the internal specific heat employed at room temperature was $1.0 .^{20}$ No temperature dependence for these last two quantities was considered. Each experimental data set was arranged as a 64element array. The convolution of the 6-moment spectrum $S(x, y)$ with the experimentally measured instrument function was evaluated in Fourier transform space by using the FFT algorithm with a 128-point zero-padded array. Zero padding was used to avoid wraparound error in the numerical convolution. Before the convolution was performed the Fou- 
rier transform of the instrument function was normalized to unity, the theoretical spectrum was normalized so as to have the same total integrated power as the experimental data, and the sampled values of $S(x, y)$ were registered with respect to the experimental data so that the locations of the centers of gravity of the theoretical spectrum and the experimental data were identical. The least-squares difference between the convolved theoretical spectrum and the experimental data was then calculated, and the values of $T$ and the density parameter $y$ producing the best two-parameter fit were determined by iteration. The 6 -moment fits to the experimental data reproduce the actual temperature to within $10 \%$ and the actual density parameter to within $20 \%$. As a check of the internal consistency of the best-fit parameters, the value of $y$ derived from the best-fit temperature by employing the ideal gas law with constant pressure $y\left(T_{\text {fit }}\right)$ was compared with the best-fit value of $y$. Ideally the ratio $y_{\mathrm{fit}} / y\left(T_{\mathrm{fit}}\right)$ should be unity. The actual values of this ratio for the 35 sets of data ranged between 0.24 and 1.07 with an average value of 0.82 . The value of the ratio was generally smaller at lower pressures $(0.2$ atm) and was generally closer to unity at higher pressures $(1.0 \mathrm{~atm})$. These small discrepancies in the fitted values of $T$ and $y$ are not altogether surprising, since the 6-moment model gives only an approximation to the actual light-scattering spectrum, since the temperature is a rapidly varying function of $x$, since $S(x, y)$ is a slowly varying function of $y$, and since the convolution with the instrument function washes out small differences in $S$ for differing $y$ values. It is currently unknown whether these discrepancies result from the approximations inherent in kinetic models for Rayleigh-Brillouin scattering themselves, ${ }^{12,20}$ represent difficulty in obtaining detailed information about the shape of the Rayleigh-Brillouin spectrum from the discretely recorded experimental spectrum, or represent an uncertainty in the determination of the half-width of the instrument function. It is also not known how noise in the experimental data affects the deconvolution process. The fitted temperatures are expected to be more accurate than the fitted densities since the density depends on the details of the shape of the RayleighBrillouin spectrum, while the temperature depends to a large extent only on the width of the spectrum, both in the $y \ll 1$ and $y \approx 1$ regions.

Returning to the fitting of the gas spectra of the static mode Fabry-Perot (i.e., imaging) experiment, we again performed the convolution of Eq. (13) in Fourier transform space, using the fast Fourier transform algorithm for only one fringe at a time with a 64-element zero-padded array for the single fringe after normalizing the instrument function to unity, normalizing the total integrated power within the theoretical scattering spectrum to the total integrated power in the experimental data for the single fringe, and registering the centers of gravity of the theoretical spectrum and the experimental data with respect to each other. The resulting least-squares deviation values of $T$ and $y$ for each separately calculated Fabry-Perot fringe in the experimental run as a function of the position of the center of the fringe are given in Figs. 5 and 6 . The computed values of $T$ and $y$ represent the spatial averages of these quantities over the area of the fringe.

The temperature profile of the flow field of Fig. 5 shows a gap in the data near the center of the Fabry-Perot interference pattern where the central fringe was too wide and too nonlinear to be usable. The hot gas jet data prominently appear at pixel $\approx 475$, and the width of the elevated temperature region agrees well with the measured inner diameter of the stainless-steel gas jet tubing. The gas jet temperature and the scattering chamber ambient temperature are somewhat lower than the readings of the thermocouples. For the ambient chamber temperature the low values of the least-squares-fitted temperatures mirror the behavior also found for the scanning mode Fabry-Perot data. We conjecture that the gas jet thermocouple reads somewhat low, since it was more than twice as far from the gas jet as the laser beam was, and some of the heat dissipated before reaching it. The temperature data for pixels 1-225, which are $\sim 9 \mathrm{~mm}$ distant from the gas jet, appear to exhibit a fluctuation that is larger than hoped for. In actuality the temperature fluctuated by no more than $\pm 10 \mathrm{~K}$ within each data set but rose steadily from the first through the seventh data set during the experimental run, which lasted $\sim 55 \mathrm{~min}$. Evidently the nitrogen in the chamber away from the gas jet began to heat. The thermocouple that was 46 $\mathrm{mm}$ downbeam was too far away to detect the slow rise.

The best-fit $y$ values are shown in Fig. 6. By comparing Figs. 5 and 6 the number density parameter data points have a much larger fluctuation than the temperature data points do. The internal consistency of the temperature and number density parameter data points was again tested by assuming the ideal gas law and constant gas pressure throughout the scattering chamber. The values of the ratio

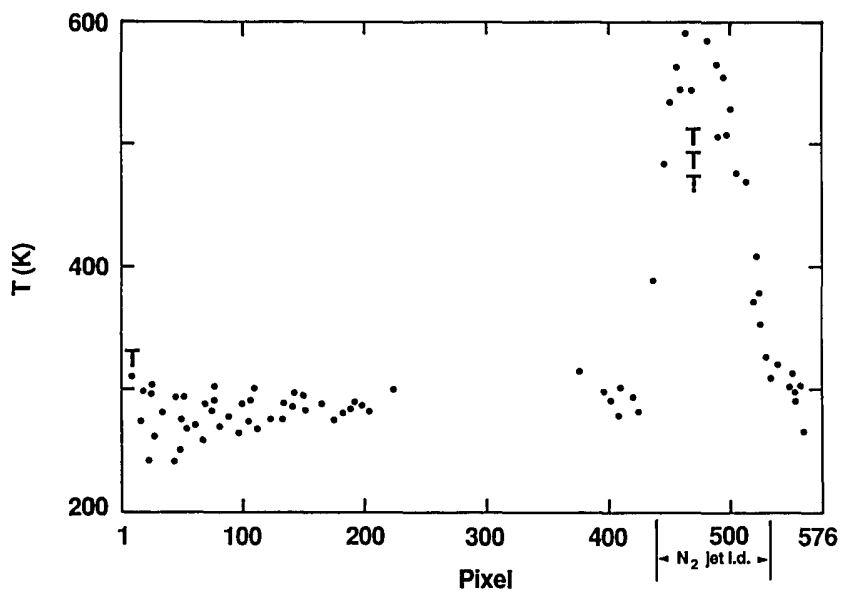

Fig. 5. Gas temperature from the least squares deviation fit as a function of pixel position. The $\mathrm{T}$ symbols are the readings of the two thermocouples of Fig. 3. 


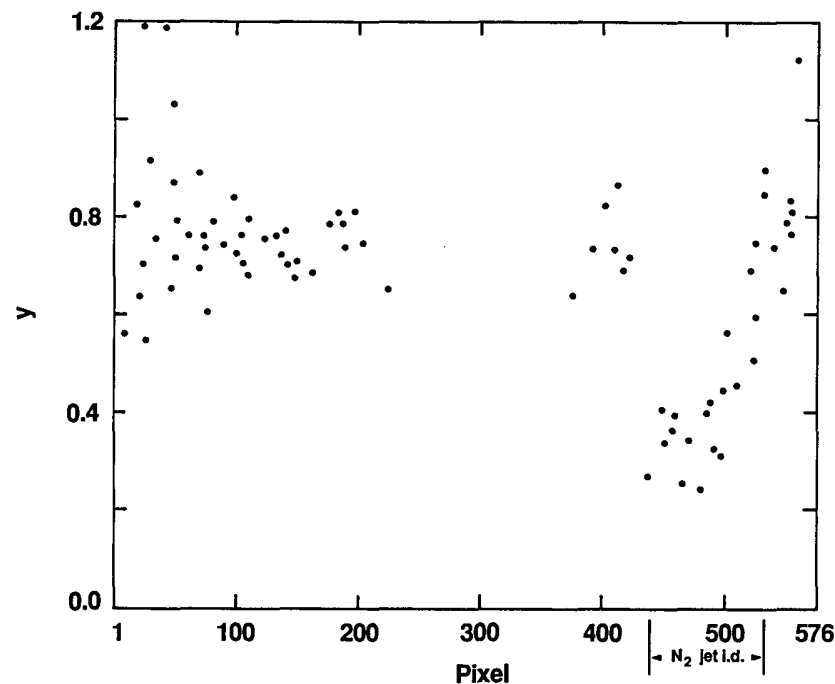

Fig. 6. Density parameter $y$ from the least-squares deviation fit as a function of pixel position.

$y_{\mathrm{fit}} / y\left(T_{\mathrm{fit}}\right)$ are given in Fig. 7. This figure again shows a systematic deviation of this ratio below the ideal value of 1.0. We believe that this deviation, along with the fluctuation of the number density data points in Fig. 6, is due to the difficulty in accurately determining the details of the shape of the Rayleigh-Brillouin spectra from the discrete CCD experimental data.

To obtain an independent measurement of the gas temperature, the absolute intensity of the RayleighBrillouin scattered light was measured and correlated to the gas number density. From this information the gas temperature was determined. The Fabry-Perot interferometer was removed from the system so that the light scattered from the laser beam at $\alpha=90^{\circ}$ was directly imaged onto the CCD array. For $T=296 \mathrm{~K}$ and four different gas pressures, the scattered light intensity was recorded for exposure times of $60 \mathrm{~s}$. The data were averaged over the seven rows of the CCD

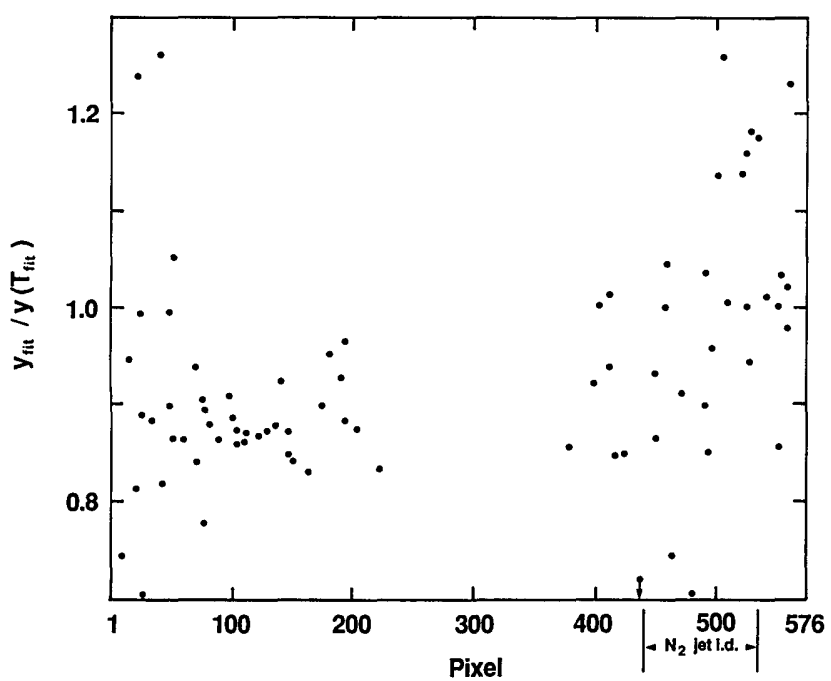

Fig. 7. Ratio of the density parameter $y$ from Fig. 6 to the density parameter obtained from the temperature of Fig. 5, the ideal gas law, and constant pressure. array corresponding to the centermost portion of the laser beam. A linear calibration curve of scattered intensity as a function of gas density was determined for each pixel in the array. The hot $\mathrm{N}_{2}$ gas jet was repositioned beneath the laser beam, and the scattered light intensity was measured at each pixel of the array. The calibration curve was then used to obtain the gas density as a function of pixel position, and the ideal gas law was used to obtain the gas temperature. The resulting temperature profile is shown in Fig. 8 along with the two thermocouple readings. The results are qualitatively similar to Fig. 5. In Fig. 8 it is seen that the thermocouple above the hot gas jet again reads low by $\sim 75 \mathrm{~K}$ and that the width of the elevated temperature region is again extended across the inner diameter of the gas jet. This additional low thermocouple reading helps confirm our conjecture that heat is dissipated between the locations of the laser beam and the gas jet thermocouple.

The results in Figs. 5 and 6 indicate that, by using a Fabry-Perot interferometer in an imaging mode, Rayleigh-Brillouin scattering has the potential to be useful as a two-dimensional combustion diagnostic, especially in the mapping of the temperature field. Of major concern, however, is the fact that the calculated temperature is a rapidly varying function of the $x$ parameter and thus is sensitive to the measured width of the instrument function. This, coupled with the requirement of imaging, represents a trade-off situation. For a detector array of fixed size with a fixed number of pixels, broadening the instrument function fringes and the gas spectra to improve their resolution results in fewer spectra appearing on the detector array and thus fewer temperature and number density data points. This also produces data points that are averaged over large spatial intervals. On the other hand, obtaining more spectra on the detector array results in narrower fringes that are

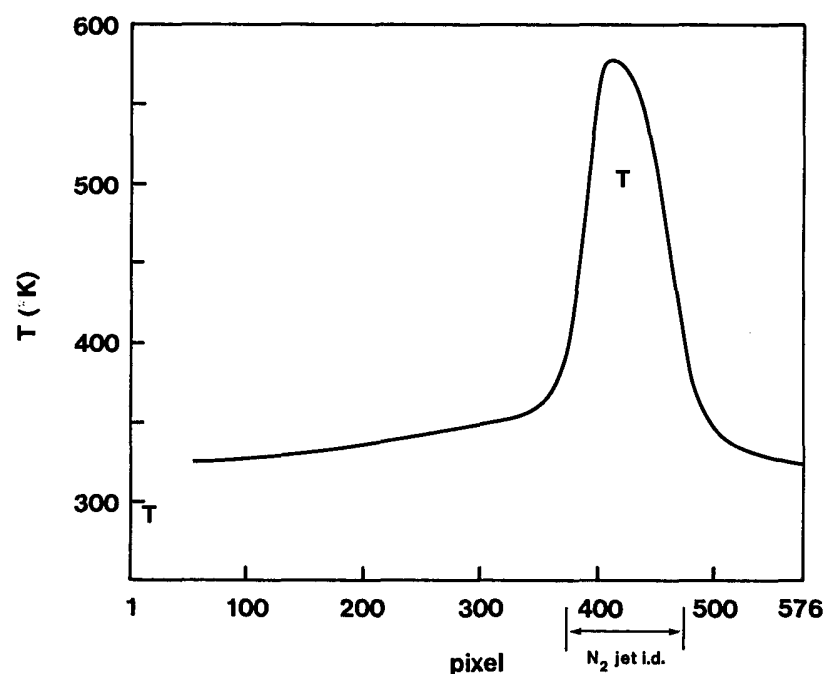

Fig. 8. Gas temperature as a function of pixel position for the repositioned heated $\mathrm{N}_{2}$ gas jet obtained by measurement of the absolute intensity of the scattered light. The $\mathrm{T}$ symbols are the readings of the two thermocouples of Fig. 3. 
difficult to resolve. We believe that our choice of $\sim 11$ usable spectra on the 576-element array is approximately the largest number that one can have and yet maintain reasonable resolvability of each spectrum. When the full width at half-maximum (FWHM) of the instrument function falls below 1 pixel, its deconvolution from the corresponding gas spectrum becomes imprecise and causes a large uncertainty in the fitted temperature. This was beginning to be the case for the data points that were near the left-hand end of the scan at pixels 15-30.

To be useful as a single-pulse combustion diagnostic, statistically reliable data must be obtained from the incident energy of a single laser pulse, i.e., $\sim 1 \mathrm{~J}$. As we mentioned above, the data of this experiment employed an incident energy of $40 \mathrm{~J}$. However, in addition to the 100-s data reported here, we also obtained reasonably stable data with integration times as low as $50 \mathrm{~s}$. We also measured the light scattered from only the central one-third of the laser beam. Better laser focusing could have resulted in the use of more of the incident laser power. Last, the vignetting resulting from the entrance and exit ports of the Fabry-Perot interferometer was more severe than the $f / 5.6$ aperture of the collection and imaging lenses, which caused an additional 'oss in usable light. These potential areas of improvement suggest that we could have obtained reasonable data with substantially less incident energy. This matter is pursued further in Appendix A. If additional refinements of the apparatus decrease the incident energy requirement further, measurement of RayleighBrillouin scattering by using a Fabry-Perot interferometer in an imaging mode could serve as a feasible combustion diagnostic, especially for temperature measurements.

\section{Appendix A: Error Estimation for Temperature Measurement by Using Two-Dimensional Rayleigh Imaging}

In this appendix we obtain an estimate of the error in the temperature that is derived from the measured Fabry-Perot fringe pattern detected with a twodimensional square pixel imaging device. For simplicity a Gaussian Rayleigh scattering spectrum, i.e., $y \ll$ 1 , is assumed. It is assumed further that the error in the actual Rayleigh spectral width is equal to the error in the measured width. Only errors that result from the assumed Poisson statistics of the detected light are considered. Thus these results represent a lower bound for the measurement error.

A single-pulse ribbon laser beam uniformly illuminates a region in the system object plane corresponding to the image of $N$ rows of detector pixels measured perpendicular to the beam propagation direction. The detector pixel size is $L_{x} \times L_{x}$, and the system has 1:1 imaging. The number of detected photons per pixel can be written as $^{37}$

$$
P=\frac{E_{p}}{N}\left(\frac{\mathrm{d} \sigma}{\mathrm{d} \Omega}\right) \mathscr{N}(\Delta \Omega) L_{x} \epsilon \frac{\lambda_{0}}{h c},
$$

where $E_{p}$ is the laser pulse energy, $\lambda_{0}$ is the laser wavelength, $(\mathrm{d} \sigma / \mathrm{d} \Omega)$ is the differential Rayleigh scattering cross section per molecule, $\mathscr{N}$ is the molecular number density, $\Delta \Omega$ is the solid angle of the detected light, $h$ is Planck's constant, $c$ is the velocity of light, and $\epsilon$ is the overall detection efficiency including detector quantum efficiency and system losses. As an example, consider nitrogen at $1 \mathrm{~atm}$ and $300 \mathrm{~K}$ giving $\mathscr{N}=2.45 \times 10^{25} \mathrm{~m}^{-3}$, a single pulse from a frequencydoubled Nd:YAG pulsed laser having $\lambda_{0}=532 \mathrm{~nm}$ and $E_{p}=1 \mathrm{~J}$, and the parameters of the experiment described in Section III, $L_{x}=23 \mu \mathrm{m}, N=384, \Delta \Omega=$ $0.016 \mathrm{sr}(f / 7), \epsilon=0.01, \mathrm{~d} \sigma / \mathrm{d} \Omega=6.1 \times 10^{-32} \mathrm{~m}^{2} / \mathrm{sr}$. Equation (A1) then gives $P=38$ detected photons per pixel per laser pulse.

Note that $P$ is the number of detected photons per pixel for the entire frequency integrated spectrum of the Rayleigh scattered light. It is the number of photons one would count without passing the light through the Fabry-Perot interferometer. The number of detected photons for light that passes through the interferometer is given by the product of the total scattered light and the instrument function of the interferometer $T(\omega, \theta)$. If the fringe-forming lens in the interferometer exit plane has focal length $f_{2}$, the radius of the fringe (which is measured from the optic axis) at the detector is $r_{0}=\theta f_{2}$. The dispersion of the interferometer is

$$
\frac{\mathrm{d} r_{0}}{\mathrm{~d} \omega}=\frac{\lambda f_{2}}{2 \pi c \theta}
$$

If we define the angular size of a single pixel as $\phi_{x}=$ $L_{x} / f_{2}$, the width in pixels of a spectral feature of width $\Delta \omega$ in $\mathrm{rad} / \mathrm{s}$ is

$$
\Delta \rho=\frac{\lambda \Delta \omega}{2 \pi c \theta \phi_{x}}
$$

Assume that the measured spectrum of the Rayleigh scattered light has the form

$$
I_{i}=a_{1} \exp \left[-\left(\rho_{i}-a_{3}\right) / a_{2}\right]^{2},
$$

where $\rho_{i}$ is the pixel index and where the peak amplitude $a_{1}$, the spectral width $a_{2}$, and peak location $a_{3}$ are unknown parameters. If we assume that the data $I_{i}$ have Poisson statistics and that there is no error in the $\rho_{i}$, prediction analysis techniques ${ }^{38}$ give the fractional uncertainty in the width $a_{2}$ :

$$
\frac{\sigma_{a_{2}}}{a_{2}}=\frac{\psi_{2}}{\left(n_{p} a_{1}\right)^{1 / 2}}
$$

where $n_{p}$ is the number of data points (pixels) in the data set, and $\psi_{2}$ is a constant that is related to the fraction of the total spectral width included in the $n_{p}$ data set. Since the temperature is proportional to $a_{2}^{2}$, the predicted fractional error in the temperature measurement is

$$
\frac{\sigma_{T}}{T}=2 \frac{\sigma_{a_{2}}}{a_{2}}
$$


If the data set to be analyzed consists of three FWHM's of the spectrum, $\psi_{2}$ attains its minimum value of $\sim 1.2$. Using a large number of data points, i.e., a wider frequency interval, does not appreciably decrease the error. For the Gaussian spectrum given by Eq. (A4), the parameter $a_{2}$ is related to the FWHM of the spectrum. This width, measured in pixels or in frequency interval, is denoted by $\Delta \rho_{\mathrm{FWHM}}$ and $\Delta \omega_{\mathrm{FWHM}}$, respectively. We have

$$
\Delta \rho_{\mathrm{FWHM}}=2 a_{2}(\ln 2)^{1 / 2} .
$$

The parameter $a_{1}$ is related to the number of counts in the pixel at the spectral peak by

$$
a_{1}=\left(\frac{\ln 2}{\pi}\right)^{1 / 2} \frac{4 \pi B P}{\Delta \omega_{\mathrm{FWHM}}},
$$

where $B$ is the instrumental bandwidth, i.e., the ratio of the interferometer free spectral range to the finesse. The number of pixels corresponding to $3 \times$ (FWHM) is thus given by Eq. (A3) as

$$
\Delta \rho_{3 \mathrm{FWHM}}=\frac{\lambda_{0} 3 \Delta \omega_{\mathrm{FWHM}}}{2 \pi c \theta \phi_{x}} .
$$

The predicted uncertainty in the temperature is then given by Eqs. (A5), (A6), (A8), and (A9):

$$
\frac{\sigma_{T}}{T}=2 \psi_{2}\left(\frac{\pi}{\ln 2}\right)^{1 / 4}\left(\frac{c \theta \phi_{x}}{6 \lambda_{0} B P}\right)^{1 / 2}
$$

As an example consider $L_{x}=23 \mu \mathrm{m}$ and $f_{2}=500 \mathrm{~mm}$ giving $\phi_{x}=0.046 \mathrm{mrad}$, a mirror spacing of $d=20$ $\mathrm{mm}$, a free spectral range of $7.5 \mathrm{GHz}$, and a finesse of 20 giving $B=375 \mathrm{MHz}$ and $P=38$ counts/pixel. The values for the fringe radii (assuming a bright central fringe), the FWHM of the Gaussian spectrum expressed in pixels, and the predicted uncertainty in the temperature measurement are given in Table I. It is important to note that these results are for a single row of pixels (i.e., using only $1 / 384$ of the total $1-\mathrm{J}$ laser energy).

A reasonable approach would be to use an approximately square region (say, 16 rows of pixels for our example), which would decrease the estimated error by a factor of 4 (e.g., to $4.6 \%$ for the third fringe). The measurement then represents an average over this

Table I. Predicted Uncertainty in Temperature Measurement of Nitrogen at STP by Using $1 / 384$ of the Height of the Region Illuminated with a 1-J Laser pulse

\begin{tabular}{cccc}
\hline $\begin{array}{c}\text { Fringe } \\
\text { Number }\end{array}$ & $\begin{array}{c}\text { Fringe } \\
\text { Radius } \\
(\mathrm{mm})\end{array}$ & $\begin{array}{c}\Delta \rho_{\text {FwHM }} \\
\text { (pixels) }\end{array}$ & $\begin{array}{c}\sigma_{T} / T \\
(\%)\end{array}$ \\
\hline 1 & 2.58 & 13.96 & 13.9 \\
2 & 3.65 & 9.87 & 16.5 \\
3 & 4.47 & 8.06 & 18.2 \\
4 & 5.16 & 6.98 & 19.6 \\
5 & 5.77 & 6.24 & 20.7 \\
6 & 6.32 & 5.70 & 21.7 \\
\hline
\end{tabular}

area. An additional way to reduce the uncertainty includes using higher laser pulse power and concentrating the laser power into a narrower strip (e.g., focusing the entire pulse energy into the 16 rows of pixels instead of the full frame of 384 rows would decrease the uncertainty by an additional factor of $384 / 16=24$ ). Where feasible, multipassing a narrow beam through the field of view could greatly increase the available power per pixel. The energy could also be increased by using multiple laser pulses, although the instantaneous nature of the measurement would be lost.

This work was carried out while J. A. Lock was a Case/NASA and NASA/American Society for Engineering Education Summer Faculty Fellow in the Optical Measurement Systems Branch of the NASA Lewis Research Center. The authors thank G. Tenti for kindly providing us with the computer code for the 6-moment model.

\section{References}

1. G. Benedek and T. Greytak, "Brillouin scattering in liquids," Proc. IEEE 53, 1623-1629 (1965).

2. A. T. Young, "Rayleigh scattering," Appl. Opt. 20, 533-535 (1981).

3. A. T. Young, "Rayleigh scattering," Phys. Today 35(1), 42-48 (1982).

4. M. Nelkin and A. Ghatak, "Simple binary collision model for Van Hove's $G_{s}(r, t)$," Phys. Rev. A 135, 4-9 (1964).

5. S. Yip and M. Nelkin, "Application of a kinetic model to time-dependent density correlations in fluids," Phys. Rev. A 135, 1241-1247 (1964).

6. S. Ranganathan and S. Yip, "Time-dependent correlations in a Maxwell gas," Phys. Fluids 9, 372-379 (1966).

7. A. Sugawara, S. Yip, and L. Sirovich, "Spectrum of density fluctuations in gases," Phys. Fluids 11, 925-932 (1968).

8. A. Sugawara, S. Yip, and L. Sirovich, "Kinetic theory analysis of light scattering in gases," Phys. Rev. 168, 121-123 (1968).

9. A. Sugawara and S. Yip, "Kinetic model analysis of light scattering by molecular gases," Phys. Fluids 10, 1911-1921 (1967).

10. C. D. Boley, R. C. Desai, and G. Tenti, "Kinetic models and Brillouin scattering in a molecular gas," Can. J. Phys. 50, 2158-2173 (1972).

11. G. Tenti, C. D. Boley, and R. C. Desai, "On the kinetic model description of Rayleigh-Brillouin scattering from molecular gases," Can. J. Phys. 52, 285-290 (1974).

12. M. Hubert and A. D. May, "The Rayleigh-Brillouin spectrum of normal and parahydrogen: A test of model solutions of the Wang-Chang Uhlenbeck equation," Can. J. Phys. 53, 343-350 (1975).

13. T. J. Greytak and G. B. Benedek, "Spectrum of light scattered from thermal fluctuations in gases," Phys. Rev. Lett. 17, 179-182 (1966).

14. E. H. Hara, A. D. May, and H. F. P. Knapp, "RayleighBrillouin scattering in compressed $\mathrm{H}_{2}, \mathrm{D}_{2}$, and HD," Can. J. Phys. 49, 420-431 (1971).

15. N. A. Clark, "Inelastic light scattering from density fluctuations in dilute gases. The kinetic-hydrodynamic transition in a monatomic gas," Phys. Rev. A 12, 232-244 (1975).

16. R. P. Sandoval and R. L. Armstrong, "Rayleigh-Brillouin spectra in molecular nitrogen," Phys. Rev. A 13, 752-757 (1976).

17. Q. H. Lao, P. E. Schoen, and B. Chu, "Rayleigh-Brillouin scattering of gases with internal relaxation," J. Chem. Phys. 64, 3547-3555 (1976). 
18. J. E. Fookson, W. S. Gornall, and H. D. Cohen, "Scaling behavior in the inert gas Brillouin spectra,"J. Chem. Phys. 65, 350-353 (1976).

19. V. Ghaem-Maghami and A. D. May, "Rayleigh-Brillouin spectrum of compressed He, Ne, and Ar. I. Scaling," Phys. Rev. A 22, 692-697 (1980).

20. A. T. Young and G. W. Kattawar, "Rayleigh-scattering line profiles," Appl. Opt. 22, 3668-3670 (1983).

21. C. Y. She, G. C. Herring, H. Moosmüller, and S. A. Lee, "Stimulated Rayleigh-Brillouin gain spectroscopy," Phys. Rev. A 31, 3733-3740 (1985).

22. R. Cattolica, F. Robben, and L. Talbot, "The interpretation of the spectral structure of Rayleigh scattered light from combustion gases," in Proceedings of the AIAA Fourteenth Aerospace Sciences Meeting, (American Institute of Aeronautics and Astronautics, New York, 1976), paper 76-31.

23. R. W. Pitz, R. Cattolica, F. Robben, and L. Talbot, "Temperature and density in a hydrogen-air flame from Rayleigh scattering," Combust. Flame 27, 313-320 (1976).

24. R. L. Schwiesow and L. Lading, "Temperature profiling by Rayleigh-scattering lidar,” Appl. Opt. 20, 1972-1979 (1981).

25. H. Shimizu, S. A. Lee, and C. Y. She, "High spectral resolution lidar system with atomic blocking filters for measuring atmospheric parameters," Appl. Opt. 22, 1373-1381 (1983).

26. H. Shimizu, K. Noguchi, and C. Y. She, "Atmospheric temperature measurement by a high spectral resolution lidar," Appl. Opt. 25, 1460-1466 (1986).

27. F. J. Lehmann, S. A. Lee, and C. Y. She, "Laboratory measurements of atmospheric temperature and backscatter ratio using a high-spectral-resolution lidar technique," Opt. Lett. 11, 563-565 (1986).

28. G. G. Sivjee, T. J. Hallinan, and G. R. Swenson, "Fabry-Perot interferometer imaging system for thermospheric temperature and wind measurements," Appl. Opt. 19, 2206-2209 (1980).

29. D. Rees, A. H. Greenway, R. Gordon, I. McWhirter, P. J. Charleton, and $\AA$. Steen, "The Doppler imaging system: initial observations of the auroral thermosphere," Planet. Space Sci. 32, 273-285 (1984).

30. V. J. Abreu and W. R. Skinner, "Inversion of Fabry-Perot CCD images: use in Doppler shift measurements," Appl. Opt. 28, 3382-3386 (1989).

31. M. Born and E. Wolf, Principles of Optics (Pergamon, New York, 1980), pp. 323-333.

32. J. M. Vaughn, The Fabry-Perot Interferometer (Hilger, London, 1989), Chap. 3.

33. G. Hernandez, Fabry-Perot Interferometers (Cambridge U. Press, Cambridge, UK, 1986), Chap. 2.5.1.

34. Reference 32, Section 3.7.

35. R. C. Weast, ed., Handbook of Chemistry and Physics (Chemical Rubber, Cleveland, Ohio, 1969), p. F-43.

36. Ref. 32, p.E-2.

37. A. C. Eckbreth, Laser Diagnostics for Combustion Temperature and Species (Abacus, Cambridge, Mass., 1988), Chap. 8.

38. J. R. Wolberg, Prediction Analysis (Van Nostrand, Princeton, N.J., 1967), Chap. 8. 